\title{
Dual-attention Guided Dropblock Module for Weakly Supervised Object Localization
}

\author{
Junhui Yin, Siqing Zhang, Dongliang Chang, Zhanyu Ma, Jun Guo \\ Beijing University of Posts and Telecommunications
}

\begin{abstract}
Attention mechanisms is frequently used to learn the discriminative features for better feature representations. In this paper, we extend the attention mechanism to the task of weakly supervised object localization (WSOL) and propose the dual-attention guided dropblock module (DGDM), which aims at learning the informative and complementary visual patterns for WSOL. This module contains two key components, the channel attention guided dropout (CAGD) and the spatial attention guided dropblock (SAGD). To model channel interdependencies, the CAGD ranks the channel attentions and treats the top- $k$ attentions with the largest magnitudes as the important ones. It also keeps some low-valued elements to increase their value if they become important during training. The SAGD can efficiently remove the most discriminative information by erasing the contiguous regions of feature maps rather than individual pixels. This guides the model to capture the less discriminative parts for classification. Furthermore, it can also distinguish the foreground objects from the background regions to alleviate the attention misdirection. Experimental results demonstrate that the proposed method achieves new state-of-the-art localization performance.
\end{abstract}

Keywords: Weakly supervised object localization, spatial attention, channel attention, dropout.

\section{Introduction}

Weakly supervised object localization (WSOL) requires less detailed annotations to identify the object location in a given image [32] compared to the fullysupervised learning. WSOL is a challenging task since neural networks have access to only image-level labels ("cat" or "no cat") that confirms the existence of the target object, but not the guidance of the expensive bounding box annotations in an image.

To address the WSOL problem with convolutional neural networks (CNNs), people resort to a general method, e.g., generating Class Activation Mapping (CAM) 32 for performing the object localization. Unfortunately, the CAM solely discovers a small part of target objects instead of the entire object, which leads to localization accuracy degradation [3].

Different from CAM, which relies only on the most discriminative information, existing approaches have explored adversarial erasing [30], Hide-and-Seek 


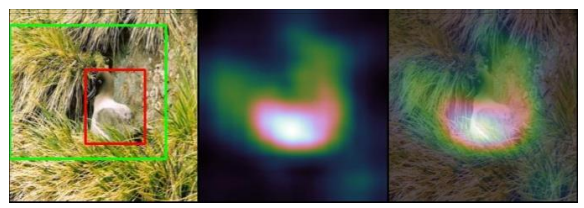

(a)

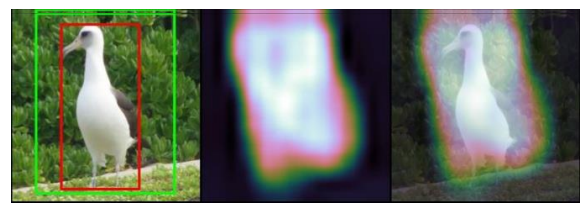

(b)

Fig. 1. Example images obtained by ResNet50-ADL. From left to right in each sub figure: the input image, the heatmap, and the overlap between the heatmap and the input image. In input image, the ground-truth bounding boxes are marked in red and the predicted are in green. The erasing operation sometimes leads to the attention spreading into the background. Meanwhile, the bounding box is too large to precisely locate the object.

(HaS) 20, Attention-based Dropout Layer (ADL) 3. Specifically, the Adversarial Complementary Learning (ACoL) approach [30] can efficiently locate different object regions and learn new and complementary parts belonging to the same objects by two adversary classifiers in a weakly supervised manner. HaS [20] hides the patches randomly, which encourages the network to seek the multiple relevant parts. ADL 3] hides the most discriminative part from the model for pursuing the full object extents and then highlights the informative parts to improve the recognition power of CNNs model. In fact, similar to the pixelbased dropout, these techniques are not really the region-based dropout that can efficiently remove the information. This is because the drop mask of ADL is generated by thresholding the pixel values on the feature map. However, the neighbouring pixels are spatially correlated on the feature map. These adjacent pixels share much of the same information. The pixel-based dropout discards the information on the convolutional feature map. However, the information are still passed on from the adjacent pixels that are left active.

Erasing the most discriminative parts is a simple yet poweful method for WSOL. For example, ADL uses the self-attention mechanism as supervision to encourage the model to learn the more useful information of the object. However, the erasing methods abandon a lot of information on the most discriminative regions. This forces the model to highlight the less discriminative parts and sometimes captures useless information of the background, which leads to the attention misdirection and the biased localization. As shown in Figure 1, the bounding box is too large to precisely locate the object, and the classification performance is not as good as before since the focused attention has been changed to other objects.

In this paper, we propose a dual-attention guided dropblock module (DGDM), a lightweight yet powerful method, for WSOL, which is illustrated in Figure 2. It contains two key components, the channel attention guided dropout (CAGD) and the spatial attention guided dropblock (SAGD), to learn the discriminative and complementary features by using the spatial and the channel attentions, respectively. Specifically, in CAGD, we first compress the spatial information 


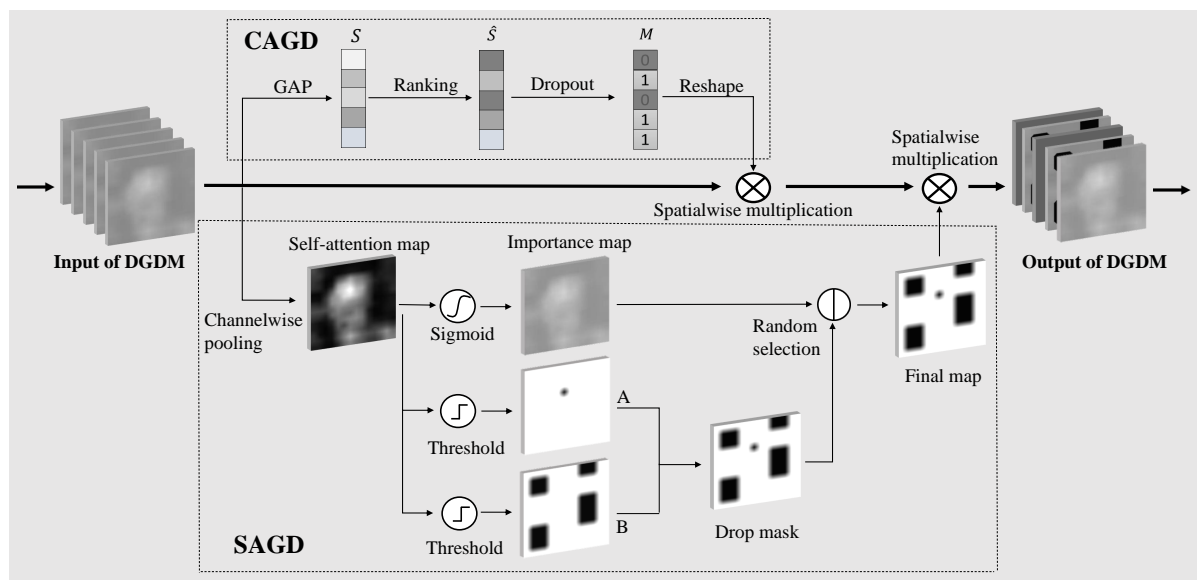

Fig. 2. Overall structure of the DGDM. It contains two key components, CAGD and SAGD. In CAGD, we rank channel attention and consider the attentions with the top-k largest magnitudes as important ones. Some low-valued elements are kept to increase their value if they become important during training. For SAGD, the drop mask can not only efficiently erase the information by removing contiguous regions of feature maps rather than individual pixels, but also sense the foreground objects and background regions to alleviate the attention misdirection. The importance map is used to highlight the most discriminative regions of target object and suppress less useful ones. Finally, we randomly select one of these two maps at each iteration and then multiply it to the input feature map. It is worth noting that this figure shows the case when the drop mask is chose.

of input feature map by GAP to generate channel attention. We also rank the obtained channel attention by a measure of importance (e.g., magnitude), and then discard some elements with low importance. In addition, some low-valued elements are also kept to increase their value if they become important during training. For SAGD, we perform channelwise average pooling on feature map to produce a self-attention map. By thresholding the obtained attention map, we generate a drop mask. It can not only efficiently erase the information by removing the contiguous regions of feature maps rather than the individual pixels, but also sense the foreground objects and the background regions under supervision with the confidence of different regions to alleviate the attention misdirection. In addition, an importance map is also generated by using a sigmoid activation on the attention map to highlight the most discriminative regions of the target object and suppress the less useful ones. We randomly select one of the two maps at each iteration and then multiply it to the input feature map.

Deep networks implemented with DGDM incorporate the image classification and WSOL. In an end-to-end learning manner, the proposed method captures the complementary and discriminative visual features for precise object localization and achieves good result of image classification.

The main contributions of the proposed method are: 
(1) We propose a lightweight and efficient attention module (DGDM) that can be easily employed to any layer of the CNNs to achieve the good performance of WSOL. The CAGD is proposed to model channel interdependencies. We rank channel attention and consider the attentions with the top- $k$ largest magnitude as important ones. We also keep some low-valued elements to increase their value if they become important during training.

(2) The SAGD is designed to generate a drop mask and an importance map. Importantly, this drop mask can efficiently erase the information by removing the contiguous regions of the feature maps and sense the foreground objects and background regions for alleviating the attention misdirection.

(3) The proposed approach can be employed to different CNNs classifiers and achieve the state-of-the-art performance on several commonly used datasets.

\section{Related Work}

Attention mechanism Attention mechanism is a data processing method learnt from human perception process [17. It does not process all the data in equal, but focuses more weights on the most informative parts [17. Attention mechanisms have demonstrated their utility across various fields, such as scene segmentation [6], image localization and understanding [13 15], finegrained visual classification [216, and image inpainting [29]. In particular, the self-attention mechanism [23] was firstly proposed to draw global dependencies of inputs and applied it in machine translation. Residual attention networks (RAN) 26] can generate attention-aware features by adopting mixed attention modules with heavy parameters. The squeeze-and-excitation module 12 was introduced to exploit the channel-interdependencies. The module can use less parameter to extract attention, and allow the network to perform feature recalibration. Convolutional block attention module 27. was proposed to emphasize the meaningful features by fusing the cross-channel and spatial information together. However, these techniques require extra training parameters for obtaining the attention map.

Dropout in convolutional neural networks Dropout 11 has been proven to be a practical technique to alleviate overfitting in fully-connected neural networks, which drops neuron activations with some fixed probability during training. All activations are used during the test phase, but the output is scaled according to the dropout probability. Many methods inspired by the original dropout have been proposed. These methods include dropconnect [25] and Monte Carlo dropout [7] and many others. However, regular dropout is less effective for convolutional layers. This can largely be attributed to two factors. The first is that convolutional layers require less regularization since these layers have much less parameters than fully-connected layers. The second factor is that there is strong correlation between the spatially adjacent pixels on the convolutional layers and these neighbouring pixels have the same information. Hence, the 
pixel-based dropout tends to abandon some information in the input, but the information can be passed on from the other adjacent pixels that are still active.

In an attempt to apply a structured form of dropout to the convolutional layer, Cutout [5] drops out contiguous regions of input images instead of individual pixels in the input layer of CNNs. This method induces the network to better utilize the contextual information of the image, rather than relying on a small set of specific features. Dropblock [8] generalizes Cutout by applying Cutout at every feature map in convolutional networks. Its main difference from regular dropout is that it discards the contiguous regions from feature map rather than independent random units. ADL [3] utilizes attention mechanism to find the maximally activated part and then drops them. However, the method only drops the strong activated pixels rather than strong activated region.

Weakly supervised object localization WSOL is an alternative cheaper way to identify the object location in a given image by solely using the imagelevel supervision, i.e., presence or absence of object categories 4321]. A WSOL method decomposes an image into a "bag" of region proposals and iteratively selecting an instance (a proposal) from each bag (an image with multiple proposals) to minimize the image classification error in step-wised manner [4. Recent research 32 utilizes CNNs classifier for specifying the spatial distribution of discriminative patterns for different image classes. A way to pursue the full object extent is self-paced learning [31. The self-produced guidance (SPG) approach utilizes the CNNs to incorporate the high confident regions, and the attention maps are then leveraged to learn the object extent under the auxiliary supervision masks of foreground and background regions. The other way to enhance the object localization is adversarial erasing [30|20]3, which first highlights the most discriminative regions on the feature map or input image and then drops them so that the less discriminative regions can be highlighted during training phase. Nevertheless, most existing approaches use alternative optimization, or requiring a lot of computing resources to erase the most discriminative regions exactly.

\section{Dual-attention guided dropblock module}

\subsection{Spatial attention module}

Let $\boldsymbol{F} \in \mathbb{R}^{H \times W \times C}$ be a convolutional feature map. Note that $C$ denotes the channel number, $H$ and $W$ are the height and the width of the feature map, respectively. For simplicity, the mini-batch dimension is omitted in this notation. We perform channelwise average pooling (CAP) on the input map $F$ to produce the 2D spatial attention map $\boldsymbol{M}_{\text {self }} \in \mathbb{R}^{H \times W}$. $\boldsymbol{M}_{\text {self }}$ is then fed into a sigmoid function to generate our importance map $\boldsymbol{M}_{i m p} \in \mathbb{R}^{H \times W}$. The spatial attention focuses on where a discriminative part is. In short, the importance map is computed as

$$
\boldsymbol{M}_{\text {self }}=C A P(\boldsymbol{F}),
$$




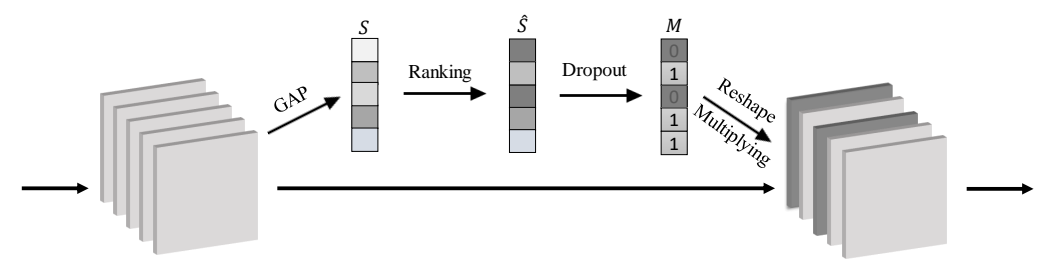

Fig. 3. Diagram of CAGD $(C=5)$. As illustrated, we first compress spatial information of a feature map by GAP to generate channel attention. We also rank channel attention according to a fast measure of importance (magnitude), and then discard some elements with low importance. Regarding to the channel selection, the binary mask is generated to indicate whether each channel is selected or not.

$$
\boldsymbol{M}_{i m p}=\sigma\left(\boldsymbol{M}_{\text {self }}\right),
$$

where $\sigma$ denotes the sigmoid function.

Convolutional layers in the model are encouraged to generate meaningful attention map for improving the classification accuracy. Therefore, in this attention map, the discriminative power of model is proportional to the intensity of each pixel. To make use of the information obtained in the CAP operation, we follow it with the second operation by using the sigmoid function, and then apply it to feature map. In this way, the spatial distribution of the most discriminative region can be approximated by this attention map efficiently, which improves the feature representation for WSOL. We observe that the importance map $M_{i m p}$ usually highlight the most discriminative regions of target object and suppress less useful ones. In particular, the most discriminative regions are some pixels whose intensity is close to one, while the parts with low values are considered as the background. Also, extra parameters are not required for our method to obtain the importance map.

\subsection{Channel attention guided dropout}

As illustrated in Figure 3, we first gather the spatial information of a feature map $\boldsymbol{F}$ by performing global average pooling (GAP) operation to generate the global information embedding: $S \in \mathbb{R}^{C}$. As mentioned before, the discriminative power of the model is proportional to the intensity of each pixel. Hence, the embedding can be considered as channel attention. According to the relative magnitude of the attention, a binary mask is generated to indicate whether each channel is selected or not. This attention-guided pruning strategies can be treated as a special way to model the interdependencies across the channels. We rank the channel attentions by a fast and approximate measure of importance (magnitude), and then discard those elements with low importance. The strategy considers the attentions with the top- $k$ largest magnitude as important ones. We also treat each element of $\boldsymbol{S}$ separately under the $L_{1}$-norm.

$$
\widehat{\boldsymbol{S}}=\left\{\arg \max _{k}\left|S_{c}\right|, S_{c} \in \boldsymbol{S}\right\}
$$




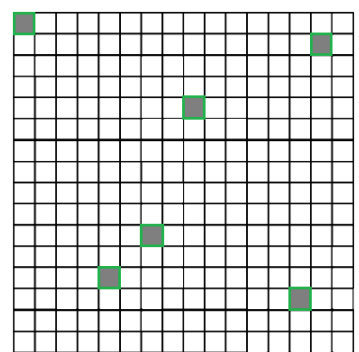

(a)

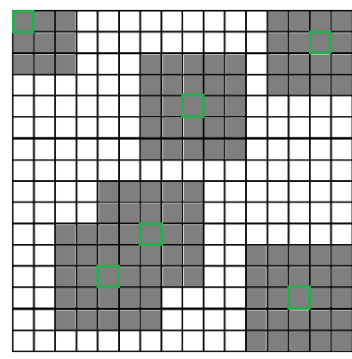

(b)

Fig. 4. (a) Original drop mask; (b) our drop mask.

where $\arg \max _{k}$ is used to return the top- $k$ elements out of all elements being considered.

Inspired by Target Dropout [9], we keep only the $k$ channels of highest magnitude in the network and drop out the others. Similar to regular dropout, this encourages the network to learn a sparse representation. However, we allow some low-valued elements to increase their value during training. As in Target Dropout 9], we introduce the stochasticity into the process using a drop probability $\alpha$ and a drop threshold $\beta$, which is the prefixed multiple of minimum value of channel attentions. Then, we obtain the drop mask $\boldsymbol{M}_{d r o p 1} \in \mathbb{R}^{C}$ by setting each element as 0 with probability $\alpha$ if it is smaller than the drop threshold $\beta$, and 1 if it is larger. To address the difficulty of learning additional parameters, we set $\alpha$ to 0.5 in our work.

\subsection{Spatial attention guided dropblock}

We observe that ADL drops only strongly activated parts according to the drop threshold. In fact, similar to the pixel-based dropout, the ADL is not really the region-based dropout. This is because the drop mask of ADL is generated by setting each pixel as 1 if it is smaller than the drop threshold $\gamma$, and 0 if it is larger. This means that $\gamma$ determines how many activation units should be discarded. However, there is the strong correlation between the neighbouring pixels on the convolutional layers, and these adjacent pixels have the similar information. Hence, the ADL cannot entirely remove the information.

To address above problems, we propose a region-based dropout in a similar fashion to regular dropout, but with an important distinction. The difference is that we drop the contiguous regions of feature maps rather than the individual pixels, as illustrated in Figure 4. Its main difference from Dropblock [8] is that its drop mask $\boldsymbol{M}_{\text {drop } 2}$ is calculated from $\boldsymbol{M}_{\text {self }}$ obtained by the spatial attention module. Then, the shared drop mask across different feature channels or each feature channel has the same drop mask. The proposed method has two main hyperparameters: block_size and $\delta$. The block_size presents the size of the block to be discarded, and $\delta$ determines how many activation units to discard. When 
block_size equals to 1 and covers the full feature map, the region-based dropout reduces to the standard dropout and SpatialDropout [22, respectively. This technique can efficiently remove the information on the feature map. Hence, it forces the network to better capture the full context of the feature map, rather than relying on the presence of a small set of the discriminative features.

It is well known that we can divide images into background and foreground regions. Also, the object of interests is usually consisted of the foreground pixels. The work 31 has reported that the attention map stands for the probabilities of corresponding pixel to be background or foreground. The initial background and object can be produced through the values in the self-attention maps. In particular, the regions with very large values are foreground, while the regions with small values are considered as background. Removing discriminative regions forces the CNNs model to capture the less discriminative part, which sometimes leads to the attention misdirection and the biased localization. Base on this, we can sense background parts and the foreground objects by using the drop mask $\boldsymbol{M}_{\text {drop } 2}$ according to the self-attention map, which will finally benefit WSOL.

We define $\boldsymbol{M}_{\text {drop } 2}$ as follows. We set $\boldsymbol{M}_{d r o p 2}^{x, y}=0$ if the pixel at $x_{t h}$ row and $y_{t h}$ column belongs to background parts or the most discriminative parts. Otherwise we have $\boldsymbol{M}_{d r o p 2}^{x, y}=1$. Specifically, $\boldsymbol{M}_{d r o p 2}$ can be calculated by

$$
\boldsymbol{M}_{\text {drop } 2}^{x, y}= \begin{cases}0, & \boldsymbol{M}_{\text {self }}^{x, y}<\delta_{l}, \boldsymbol{M}_{\text {self }}^{x, y}>\delta_{h}, \\ 1, & \text { otherwise },\end{cases}
$$

where $\delta_{l}$ and $\delta_{h}$ are thresholds to identify the regions in feature maps as the background and the most discriminative parts of foreground, respectively.

\subsection{Network Implementation}

Fully CNNs with DGDM fuse the complementary discriminative parts for precise object localization and the accurate image classification in an end-to-end manner. Following the work in [3], the DGDM is inserted in higher-level feature maps of the CNNs model.

For the DGDM, convolutional feature maps are averaged by channelwise average pooling to generate the meaningful self-attention map. The attention maps are then activated by using a sigmoid function. We then multiply the obtained importance map to input. Based on this attention map, the CNNs model can distinguish the foreground objects from background regions by multiplying $\boldsymbol{M}_{\text {drop } 2}$ to the input feature map. The importance map $\boldsymbol{M}_{i m p}$ highlights the most discriminative information for achieving the good accuracy of the model. Unfortunately, the classifiers often rely only on the most discriminative information. The work in 20 has reported that losing some classification accuracy results in the huge boost in localization performance. This is owing to the application of a drop mask, which erases the most discriminative parts. From this idea, the importance map or drop mask is stochastically selected during training phase. As presented in ADL [3, we also introduce a drop rate, which controls how frequently the drop mask is employed. In addition, channel attention guided mask 
$\boldsymbol{M}_{\text {drop } 1}$ is also applied to input feature maps to model the interdependencies across the channels.

The CNNs model is optimized with Stochastic Gradient Descent (SGD) algorithm. We extract the heatmap using the same method as introduced in [32]. Finally, a thresholding method [38] is then directly applied to predict the locations of target object.

\section{Experiment}

\subsection{Experimental Setup}

Datasets We evaluate the performance of the proposed method in the commonly used CUB-200-2011 [24, Stanford Cars [14, and ILSVRC 2016 [18] datasets.

Evaluation metrics Three evaluation metrics are used for WSOL evaluation [20. They are localization accuracy with known ground-truth class (GT-Loc), Top-1 classification accuracy (Top-1 Clas), and Top-1 localization accuracy (Top1 Loc). It is worth noting that the most appropriate metric is Top-1 Loc for evaluating the performance of WSOL.

Experimental details The proposed DGDM is integrated with the commonly used CNNs including VGG [19], ResNet [10, ResNet-SE [12], and InceptionV3 21. Following the settings of work [3, the drop rate is set as $75 \%$, and apply DGDM to higher-level and intermediate layers of CNNs. These networks are initialized with ImageNet pre-trained weights.

\subsection{Ablation studies}

The ablation studies on CUB-200-2011 with the pre-trained VGG-GAP are used to evaluate the effects of the proposed DGDM. Furthermore, we conduct some experiments on Stanford Cars to investigate the effect of network depth on localization performance. During training phase, the DGDM is inserted in all the pooling layers and the conv5-3 layer.

The drop mask map $\boldsymbol{M}_{\text {drop } 2}$ can not only remove a small set of the discriminative features to better capture the full context of the feature map, but also identify the regions in the feature maps as the background and the foreground, respectively. First, we verify the effectiveness of removing importance parts on accuracy. The upper block of Table 1 presents the experimental results when different block_size are adopted. From these results, it can be seen that we achieve the best localization accuracy when block_size is 2 . We also present the result when block_size is adaptive and calculated by $[\mathrm{H}(\mathrm{W}) / 7]$. It can be observed that the drop masks with block_size $=2$ remove the discriminative region 
Table 1. Upper and Middle: Accuracy according to different block_size. Lower: Accuracy according to different drop threshold $\beta$. $\boldsymbol{M}_{\text {drop2_stage1: eras- }}$ ing the most discriminative parts of the object. $\boldsymbol{M}_{\text {drop 2_stage1 }} \boldsymbol{M}_{\text {drop 2_stage2: }}$ removing the most discriminative parts and alleviating attention misdirection. $\boldsymbol{M}_{\text {drop } 2 \text { stage1 }}+\boldsymbol{M}_{\text {drop } 2 \_s t a g e 2}+\boldsymbol{M}_{\text {drop } 1}$ : removing the most discriminative parts of the object, alleviating attention misdirection, and utilizing channel attention guided dropout. Adap_7: adaptive and block_size calculated by $[\mathrm{H}(\mathrm{W}) / 7]$.

\begin{tabular}{|c|c|c|c|c|}
\hline \multirow[t]{3}{*}{ Method } & \multicolumn{2}{|c|}{ block_size GT } & \multirow{2}{*}{$\begin{array}{l}\text { Top-1 } \\
\text { Clas }(\%)\end{array}$} & \multirow{2}{*}{$\begin{array}{l}\text { Top-1 } \\
\text { Loc }(\%)\end{array}$} \\
\hline & & $\operatorname{Acc}(\%)$ & & \\
\hline & 1 & 62.70 & 71.99 & 45.20 \\
\hline \multirow[t]{3}{*}{$M_{d r o p 2 \_s t a g e 1}$} & 2 & 73.48 & 68.30 & 52.57 \\
\hline & 3 & 69.53 & 55.56 & 43.10 \\
\hline & Adap_7 & 73.52 & 65.83 & 50.46 \\
\hline \multirow{5}{*}{$\boldsymbol{M}_{d r o p 2 \_ \text {stage } 1+M_{d r o p 2 \_s t a g e} 2}$} & 1 & 73.48 & 69.11 & 52.11 \\
\hline & 2 & 73.94 & 69.68 & 53.27 \\
\hline & 3 & 74.23 & 69.00 & 53.79 \\
\hline & 4 & 73.48 & 69.11 & 52.11 \\
\hline & Adap_7 & 72.02 & 69.21 & 51.42 \\
\hline \multirow{6}{*}{ 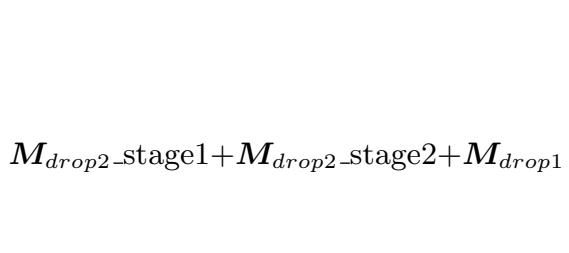 } & $\beta$ & GT & Top-1 & Top-1 \\
\hline & & $\operatorname{Acc}(\%)$ & Clas (\%) & $\operatorname{Loc}(\%)$ \\
\hline & 2 & 73.44 & 69.19 & 52.56 \\
\hline & 2.5 & 74.88 & 69.50 & 54.31 \\
\hline & 3 & 75.02 & 69.89 & 54.34 \\
\hline & 3.5 & 72.65 & 69.68 & 52.67 \\
\hline
\end{tabular}

more precisely than those with other block_size. We observe that the classification accuracy decreases as block_size increases. This is because the model never captures the most discriminative region.

Next, we investigate the effect of removing a small set of background on accuracy. The middle block of Table 1 presents the results when we use different block_size. It can be observed that the best object localization accuracy can be established when block_size is 3. In addition, the Top-1 Loc increases again (from $52.57 \%$ to $53.79 \%$ ) and the classification accuracy achieve $0.7 \%$ improvement (from $68.30 \%$ to $69.00 \%$ ). This indicates that removing a small set of background boosts the performance of WSOL. The reason lies in that the proposed erasing method does not lead to the attention misdirection when the discriminative parts are erased.

Thirdly, we observe the effect of channel attention guided dropout on the accuracy by using four different drop thresholds. The lower block of Table 1 reports experimental results. Based on this, we can conclude that the value of the drop threshold has an important effect on performance of WSOL. It can also be seen that three evaluation metrics are improved when the drop threshold is 3 . 
Table 2. Performance comparison on Stanford Cars test set with several popular baseline architectures.

\begin{tabular}{cccc}
\hline \hline \multicolumn{4}{c}{ Method Backbone Top-1 Loc Top-1 Clas } \\
\hline ADL & ResNet18 & 86.50 & 88.50 \\
Ours & ResNet18 & $\mathbf{8 7 . 3 8}$ & 88.91 \\
\hline ADL & ResNet34 & 87.37 & 90.24 \\
Ours & ResNet34 & $\mathbf{8 8 . 4 4}$ & 89.91 \\
\hline ADL & ResNet50 & 89.32 & 91.48 \\
Ours & ResNet50 & $\mathbf{9 0 . 2 5}$ & 91.85 \\
\hline \hline
\end{tabular}

Lastly, we generalise our method to other dataset (Stanford Cars) and investigate the effect of network depth on localization accuracy. Some experiments are performed with several popular baseline architectures (ResNet-18, ResNet34, ResNet-50). DGDM is integrated into these networks. This method and its counterpart (ADL) are trained on the Stanford Cars dataset. We report the performance of our method and its counterpart on Stanford Cars in Table 2. It can be observed that in all the comparisons the proposed method outperforms its counterpart and the proposed method obtains the best performance when ResNet-50 is employed as backbone. This suggests that the benefits of DGDM are not limited to network depth and certain dataset.

\subsection{Comparison with the state-of-the-arts}

We compare our proposed approach with existing WSOL techniques on the CUB200-2011 test set and ILSVRC validation set and give the results in Table 3. Table 4 and Table 2, respectively. These approaches include CAM [32, DANet [28], $\mathrm{ACoL}$ 30], SPG 31, and ADL [3].

CUB-200-2011 Table 3 summaries the quantitative evaluation results on the CUB-200-2011 test set. With a VGG-GAP backbone, our method reports $4.58 \%$ higher Top-1 Clas and 1.98\% higher Top-1 Loc compared with the ADL approach [3]. With a ResNet50 backbone, it reports $8.30 \%$ performance gain over the DANet approach [28] at the cost of little classification performance. This is the outcome of the trade-off relationship between classification and localization accuracy discussed in Subsection 3.4. Our method significantly outperforms all the existing approaches, and obtains a new SOTA localization accuracy $(59.40 \%)$ even when other two backbones are used. When InceptionV3 is used as a backbone, this method still has comparable accuracy.

In addition to obtaining a better performance of WSOL, this method has high efficiency. Table 3 also presents the number of parameters as well as parameter and computation overheads. Similar to ADL [3], additional training parameters are not required for our method, and there are no computation overheads upon the backbone network. 
Table 3. Quantitative evaluation results on CUB-200-2011 test set with the state-ofthe-art results.

\begin{tabular}{|c|c|c|c|c|c|c|c|}
\hline \multirow[t]{2}{*}{ Method } & \multirow[t]{2}{*}{ Backbone } & \multirow{2}{*}{$\begin{array}{c}\text { FLOPs } \\
(\mathrm{Gb})\end{array}$} & \multirow{2}{*}{$\begin{array}{c}\text { \# of } \\
\text { Params } \\
(\mathrm{Mb})\end{array}$} & \multicolumn{2}{|c|}{ Overheads } & \multicolumn{2}{|c|}{ CUB-200-2011 } \\
\hline & & & & Computation(\%) & Parameter $(\%)$ & $\begin{array}{l}\text { Top-1 } \\
\text { Loc }(\%)\end{array}$ & $\begin{array}{l}\text { Top-1 } \\
\text { Clas }(\%)\end{array}$ \\
\hline CAM & VGG-GAP & 18.20 & 29.08 & 0 & 0 & 34.41 & 67.55 \\
\hline $\mathrm{ACoL}$ & VGG-GAP & 31.98 & 37.63 & 71.51 & 75.71 & 45.92 & 71.90 \\
\hline ADL & VGG-GAP & 18.20 & 29.08 & 0 & 0 & 52.36 & 65.27 \\
\hline DANet & VGG-GAP & 24.12 & 48.56 & 32.53 & 66.99 & 52.52 & 75.40 \\
\hline Ours & VGG-GAP & 18.20 & 29.08 & 0 & 0 & 54.34 & 69.85 \\
\hline $\mathrm{ADL}$ & ResNet50 & 62.32 & 23.92 & 0 & 0 & 46.29 & 79.72 \\
\hline DANet & ResNet50 & 74.33 & 32.63 & 19.27 & 36.41 & 51.10 & 81.60 \\
\hline Ours & ResNet50 & 62.32 & 23.92 & 0 & 0 & 59.40 & 76.20 \\
\hline CAM & InceptionV3 & 4.84 & 25.69 & 0 & 0 & 43.67 & - \\
\hline $\mathrm{SPG}$ & InceptionV3 & 31.98 & 37.63 & 560.74 & 46.48 & 46.64 & - \\
\hline ADL & InceptionV3 & 4.84 & 25.69 & 0 & 0 & 53.04 & 74.55 \\
\hline DANet & InceptionV3 & 7.23 & 30.62 & 49.38 & 18.47 & 49.45 & 71.20 \\
\hline Ours & InceptionV 3 & 4.84 & 25.69 & 0 & 0 & 52.62 & 72.23 \\
\hline
\end{tabular}

Table 4. Quantitative evaluation results on ILSVRC validation set with the state-ofthe-art results.

\begin{tabular}{cccc}
\hline \hline Method & Backbone & Top-1 Loc Top-1 Clas \\
\hline CAM & ResNet50-SE & 46.19 & 76.56 \\
ADL & ResNet50-SE & 48.53 & 75.56 \\
Ours & ResNet50-SE & $\mathbf{4 8 . 8 1}$ & 73.50 \\
\hline \hline
\end{tabular}

ILSVRC In Table 4, we evaluate the performance of our proposed method on the large-scale ILSVRC dataset. With a ResNet50-SE backbone, our method obtains a better localization performance than ADL and CAM. To sum up, our method achieves new state-of-the-art accuracy compared with the current techniques.

Discussion The work in 3 has reported that ADL extracts the discriminative information of the background that often appears with the object. To investigate the substantial difference between the proposed method and ADL, we show the heatmaps as well as the predicted bounding boxes on Stanford Cars and CUB-200-2011 in Figure 5. It can be observed that the object localization maps produced by our method can obtain more accurate bounding boxes than ADL. That is, the network implemented with DGDM learns well to exploit the information in the target object regions and learn patterns from them. We attribute it to the removing operation which induces the network to discover more discriminative features to achieve better performance of WSOL. On the CUB-200-2011 

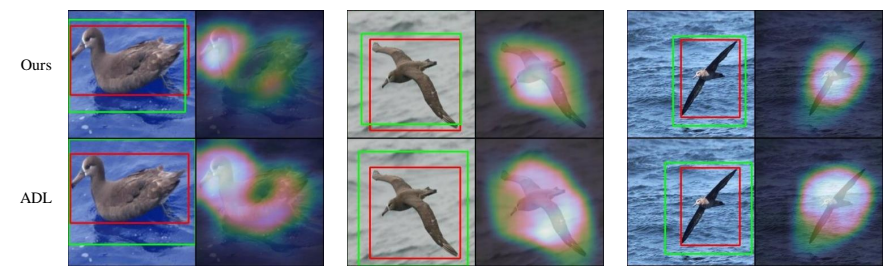

(a) CUB-200-2011
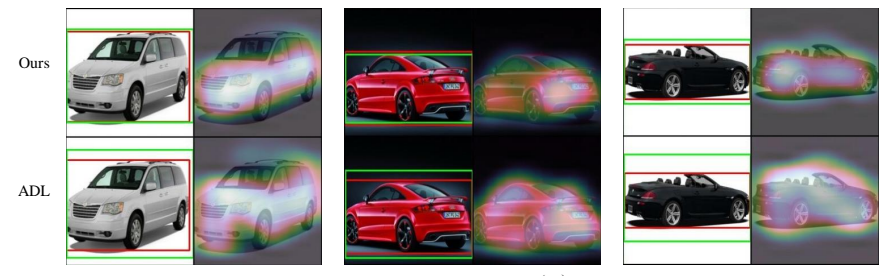

(b) Stanford Cars
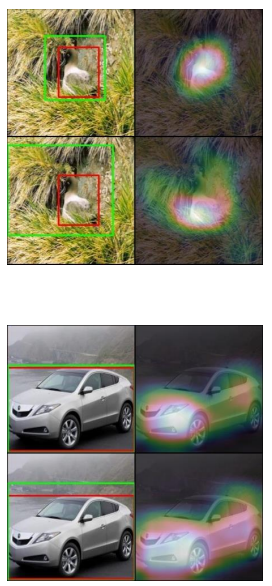

Fig. 5. Visualization results of ResNet50 on CUB-200-2011 and Stanford Cars. We compare the visualization results of DGDM-integrated network (ResNet50 + DGDM) with ADL. From left image and right image in each figure: input image and the overlap between the heatmap and the input image. In input image, the ground-truth bounding boxes are in red and the predicted are in green.

dataset, the less discriminative part sometimes is the background frequently occurring with the object 3 . For example, our method and ADL can discover nearly entire parts of a bird, e.g., the wing and head, but ADL also learns more background information when the most discriminative information is discarded.

\section{Conclusions}

In this paper, we proposed a simple yet effective dual-attention guided dropblock module (DGDM) for weakly supervised object localization (WSOL). We designed two key components of DGDM, namely the channel attention guided dropout (CAGD) and the spatial attention guided dropblock (SAGD), and integrated them with the deep learning framework. The proposed method hides the most discriminative part and then encourages the CNNs model to discover the less discriminative part. We defined a pruning strategy so that CAGD can be adapted to model the interdependencies across the channels. In addition, SAGD can not only efficiently remove the information by erasing the contiguous regions of feature maps rather than the independent individual pixels, but also sense the target objects and background regions to alleviate the attention misdirection. Compared to some existing WSOL techniques, the proposed method is lightweight, and can be easily employed to different CNNs classifiers. We also have achieved new SOTA localization accuracy on CUB-200-2011, Stanford Cars, and ILSVRC. 


\section{References}

1. Bency, A.J., Kwon, H., Lee, H., Karthikeyan, S., Manjunath, B.: Weakly supervised localization using deep feature maps. In: Proceedings of the European Conference on Computer Vision (ECCV). pp. 714-731. Springer (2016)

2. Chang, D., Ding, Y., Xie, J., Bhunia, A.K., Li, X., Ma, Z., Wu, M., Guo, J., Song, Y.Z.: The devil is in the channels: Mutual-channel loss for fine-grained image classification. IEEE Transactions on Image Processing 29, 4683-4695 (2020)

3. Choe, J., Shim, H.: Attention-based dropout layer for weakly supervised object localization. In: Proceedings of the IEEE Conference on Computer Vision and Pattern Recognition (CVPR). pp. 2219-2228 (2019)

4. Cinbis, R.G., Verbeek, J., Schmid, C.: Weakly supervised object localization with multi-fold multiple instance learning. IEEE Transactions on Pattern Analysis and Machine Intelligence 39(1), 189-203 (2016)

5. DeVries, T., Taylor, G.W.: Improved regularization of convolutional neural networks with cutout. arXiv preprint arXiv:1708.04552 (2017)

6. Fu, J., Liu, J., Tian, H., Li, Y., Bao, Y., Fang, Z., Lu, H.: Dual attention network for scene segmentation. In: Proceedings of the IEEE Conference on Computer Vision and Pattern Recognition (CVPR). pp. 3146-3154 (2019)

7. Gal, Y., Ghahramani, Z.: Dropout as a bayesian approximation: Representing model uncertainty in deep learning. In: International Conference on Machine Learning (ICML). pp. 1050-1059 (2016)

8. Ghiasi, G., Lin, T.Y., Le, Q.V.: Dropblock: A regularization method for convolutional networks. In: Advances in Neural Information Processing Systems (NIPS). pp. 10727-10737 (2018)

9. Gomez, A.N., Zhang, I., Swersky, K., Gal, Y., Hinton, G.E.: Targeted dropout

10. He, K., Zhang, X., Ren, S., Sun, J.: Deep residual learning for image recognition. In: Proceedings of the IEEE Conference on Computer Vision and Pattern Recognition (CVPR). pp. 770-778 (2016)

11. Hinton, G.E., Srivastava, N., Krizhevsky, A., Sutskever, I., Salakhutdinov, R.R.: Improving neural networks by preventing co-adaptation of feature detectors. arXiv preprint arXiv:1207.0580 (2012)

12. $\mathrm{Hu}$, J., Shen, L., Sun, G.: Squeeze-and-excitation networks. In: Proceedings of the IEEE Conference on Computer Vision and Pattern Recognition (CVPR). pp. $7132-7141$ (2018)

13. Jaderberg, M., Simonyan, K., Zisserman, A., et al.: Spatial transformer networks. In: Advances in Neural Information Processing Systems (NIPS). pp. 2017-2025 (2015)

14. Krause, J., Stark, M., Deng, J., Fei-Fei, L.: 3d object representations for finegrained categorization. In: Proceedings of the IEEE International Conference on Computer Vision Workshops. pp. 554-561 (2013)

15. Li, K., Wu, Z., Peng, K.C., Ernst, J., Fu, Y.: Tell me where to look: Guided attention inference network. In: Proceedings of the IEEE Conference on Computer Vision and Pattern Recognition (CVPR). pp. 9215-9223 (2018)

16. Li, X., Chang, D., Ma, Z., Tan, Z.H., Xue, J.H., Cao, J., Yu, J., Guo, J.: Oslnet: Deep small-sample classification with an orthogonal softmax layer. IEEE Transactions on Image Processing (2020)

17. Mnih, V., Heess, N., Graves, A., et al.: Recurrent models of visual attention. In: Advances in Neural Information Processing Systems (NIPS). pp. 2204-2212 (2014) 
18. Russakovsky, O., Deng, J., Su, H., Krause, J., Satheesh, S., Ma, S., Huang, Z., Karpathy, A., Khosla, A., Bernstein, M., et al.: Imagenet large scale visual recognition challenge. International Journal of Computer Vision 115(3), 211-252 (2015)

19. Simonyan, K., Zisserman, A.: Very deep convolutional networks for large-scale image recognition. arXiv preprint arXiv:1409.1556 (2014)

20. Singh, K.K., Lee, Y.J.: Hide-and-seek: Forcing a network to be meticulous for weakly-supervised object and action localization. In: 2017 IEEE International Conference on Computer Vision (ICCV). pp. 3544-3553. IEEE (2017)

21. Szegedy, C., Vanhoucke, V., Ioffe, S., Shlens, J., Wojna, Z.: Rethinking the inception architecture for computer vision. In: Proceedings of the IEEE Conference on Computer Vision and Pattern Recognition (CVPR). pp. 2818-2826 (2016)

22. Tompson, J., Goroshin, R., Jain, A., LeCun, Y., Bregler, C.: Efficient object localization using convolutional networks. In: Proceedings of the IEEE Conference on Computer Vision and Pattern Recognition (CVPR). pp. 648-656 (2015)

23. Vaswani, A., Shazeer, N., Parmar, N., Uszkoreit, J., Jones, L., Gomez, A.N., Kaiser, Ł., Polosukhin, I.: Attention is all you need. In: Advances in Neural Information Processing Systems (NIPS). pp. 5998-6008 (2017)

24. Wah, C., Branson, S., Welinder, P., Perona, P., Belongie, S.: The caltech-ucsd birds-200-2011 dataset (2011)

25. Wan, L., Zeiler, M., Zhang, S., Le Cun, Y., Fergus, R.: Regularization of neural networks using dropconnect. In: International Conference on Machine Learning (ICML). pp. 1058-1066 (2013)

26. Wang, F., Jiang, M., Qian, C., Yang, S., Li, C., Zhang, H., Wang, X., Tang, X.: Residual attention network for image classification. In: Proceedings of the IEEE Conference on Computer Vision and Pattern Recognition (CVPR). pp. 3156-3164 (2017)

27. Woo, S., Park, J., Lee, J.Y., So Kweon, I.: Cbam: Convolutional block attention module. In: Proceedings of the European Conference on Computer Vision (ECCV). pp. 3-19 (2018)

28. Xue, H., Liu, C., Wan, F., Jiao, J., Ji, X., Ye, Q.: Danet: Divergent activation for weakly supervised object localization. In: Proceedings of the IEEE International Conference on Computer Vision (ICCV). pp. 6589-6598 (2019)

29. Yu, J., Lin, Z., Yang, J., Shen, X., Lu, X., Huang, T.S.: Generative image inpainting with contextual attention. In: Proceedings of the IEEE Conference on Computer Vision and Pattern Recognition (CVPR). pp. 5505-5514 (2018)

30. Zhang, X., Wei, Y., Feng, J., Yang, Y., Huang, T.S.: Adversarial complementary learning for weakly supervised object localization. In: Proceedings of the IEEE Conference on Computer Vision and Pattern Recognition (CVPR). pp. 1325-1334 (2018)

31. Zhang, X., Wei, Y., Kang, G., Yang, Y., Huang, T.: Self-produced guidance for weakly-supervised object localization. In: Proceedings of the European Conference on Computer Vision (ECCV). pp. 597-613 (2018)

32. Zhou, B., Khosla, A., Lapedriza, A., Oliva, A., Torralba, A.: Learning deep features for discriminative localization. In: Proceedings of the IEEE Conference on Computer Vision and Pattern Recognition (CVPR). pp. 2921-2929 (2016) 